\title{
TMEM173 Gene
}

National Cancer Institute

\section{Source}

National Cancer Institute. TMEM173 Gene. NCI Thesaurus. Code C101586.

This gene plays a role in the immune response. 